\title{
Application of MZI Symmetrical Structure With Fiber Balls and Seven-Core Fiber in Microdisplacement Measurement
}

\author{
Liming $\mathrm{ZHAO}^{1}$, Hong $\mathrm{LI}^{1,3}$, Yanming $\mathrm{SONG}^{1}$, Mingli $\mathrm{DONG}^{2 *}$, and Lianqing $\mathrm{ZHU}^{1,3^{*}}$ \\ ${ }^{1}$ Beijing Laboratory of Optical Fiber Sensing and System, Beijing Information Science \& Technology University, Beijing \\ 100016, China \\ ${ }^{2}$ Beijing Key Laboratory of Optoelectronic Measurement Technology, Beijing Information Science \& Technology \\ University, Beijing 100192, China \\ ${ }^{3}$ Overseas Expertise Introduction Center for Discipline Innovation ("111 Center"), Beijing Information Science \& \\ Technology University, Beijing 100192, China
}

${ }^{*}$ Corresponding authors: Mingli DONG and Lianqing ZHU

E-mail: dongml@sina.com and lqzhu_bistu@sina.com

\begin{abstract}
An optical fiber microdisplacement sensor based on symmetric Mach-Zehnder interferometer (MZI) with a seven-core fiber and two single-mode fiber balls is proposed. The rationality and manufacturing process of the MZI sensing structure are analyzed. The fabrication mechanism of the Mach-Zehnder sensor by $\mathrm{CO}_{2}$ laser is described in detail. Experimental results show that temperature sensitivities of the two dips are $98.65 \mathrm{pm} /{ }^{\circ} \mathrm{C}$ and $89.72 \mathrm{pm} /{ }^{\circ} \mathrm{C}$, respectively. The microdisplacement sensitivities are $2017.71 \mathrm{pm} / \mathrm{mm}$ and $2457.92 \mathrm{pm} / \mathrm{mm}$, respectively. The simultaneous measurement of temperature and microdisplacement is demonstrated based on the sensitive matrix. The proposed Mach-Zehnder interference sensor exhibits the advantages of compact structure, simple manufacturing process, and high reliability.
\end{abstract}

Keywords: Microdisplacement; Mach-Zehnder interferometer; fiber ball; symmetrical structure; seven-core fiber

Citation: Liming ZHAO, Hong LI, Yanming SONG, Mingli DONG, and Lianqing ZHU, "Application of MZI Symmetrical Structure With Fiber Balls and Seven-Core Fiber in Microdisplacement Measurement," Photonic Sensors, 2019, 9(2): 97-107.

\section{Introduction}

Microdisplacement sensors have wide applications in automatic equipment, robots, and road and bridge management [1-6]. High precision displacement sensors include the inductive displacement sensor [2], capacitive displacement sensor [3], photoelectric displacement sensor [4, 5], ultrasonic displacement sensor [6], and so on. With the development of optical fiber technology, many optical fiber displacement sensors have emerged. Generally, the optical fiber sensor has many advantages, such as small size, light weight, electromagnetic immunity, and good link-reusability [7]. On the basis of the above advantages, the optical fiber displacement sensor has quickly won the favor of many researchers.

Fiber sensor could solve many problems of the displacement measurement in severe environment. In 2012, Zhong et al. [8] designed the fiber Bragg grating (FBG) displacement sensor which is based on the double-trapezoidal cantilever beam structure with the displacement sensitivity of $0.25 \mathrm{~nm} / \mathrm{mm}$. In addition to the existing cantilever beam structure, the deformation ring structure is also a hot research topic. Compared with cantilever beam structure, it

Received: 22 July 2018 / Revised: 20 September 2018

(C) The Author(s) 2018. This article is published with open access at Springerlink.com

DOI: $10.1007 / \mathrm{s} 13320-018-0518-5$

Article type: Regular 
has higher measuring precision. In 2018, Shangguan et al. [9] designed an optical fiber Fabry-Perot sensor based on hydrofluoric acid corrosion, and the displacement sensitivity of the sensor can reach $0.0100 \mathrm{~nm} / \mu \mathrm{m}$ within the range of $0 \mu \mathrm{m}-1000 \mu \mathrm{m}$ displacement at room temperature. As a kind of fiber optic sensor, Mach-Zehnder interferometer (MZI) fiber optic sensor has the characteristics of simple structure, portability, easy manufacture, high sensitivity, and large sensing wavelength range. MZ interference micro-displacement sensor has been widely studied [10-12]. In 2013, Chen et al. [13] proposed a micro-displacement sensor with a core-offset structure. Its microdisplacement sensitivity is $0.835 \mathrm{~nm} / \mu \mathrm{m}$. The core-offset structure is very sensitive to temperature, so the experimental result is easily affected by temperature. In 2013, Shen et al. [14] put forward a microdisplacement sensor based on an MZI made of a bowknot type taper. Experimental results show that the microdisplacement sensitivity is $1.93 \mathrm{~dB} / \mu \mathrm{m}$ and the temperature sensitivity is $0.023 \mathrm{~dB} /{ }^{\circ} \mathrm{C}$, so the cross-sensitivity effect on the proposed sensor can be neglected in practical applications. But it cannot realize the measurement of temperature and microdisplacement simultaneously. In recent researches, the fiber Bragg grating has been combined with an MZI in order to achieve the simultaneous measurement of temperature and micro displacement (or strain and other parameters). In 2017, Zhou et al. [15] proposed a single-mode and multimode temperature sensing structure which can measure temperature $800{ }^{\circ} \mathrm{C}$ accurately. Compared with the traditional sensor, the sensitivity and the measuring range have been greatly improved. The structure needs to reasonably control the multi-mode optical fiber welding parameters, higher requirements for machining process and the displacement measurement which has not been realized.

In this paper, an optical fiber microdisplacement sensor based on symmetric Mach-Zehnder interferometer with a seven-core fiber and two single-mode fiber balls is proposed. Based on its good sensing characteristics and good response sensitivity, the sensor has great potential in the direction of microdisplacement measurement. In Section 2, the rationality of the sensing structure is analyzed. Moreover, the manufacturing process and sensing principle of the sensing structure are described in detail. The role of the sensing structure in MZI sensing is also explained. In Section 3, the sensor sensing characteristic experiment, including temperature characteristic test and microdisplacement characteristic test, is completed. The experimental results are analyzed as well. In Section 4 , a discussion and some conclusions are finally made.

\section{Sensor structure and principle}

\subsection{Sensor design}

We have spliced the seven-core fiber (SCF) with different lengths between two segments of single mode fibers (SMFs) to construct the SMF-SCF-SMF structure (i.e. without two fiber balls). However, the interferometer can't be formed or the response is weak. Figure 1 shows the transmission spectra of different structure MZIs.

As shown in Fig. 1(a), the purple line is the spectrum of the MZI with $5 \mathrm{~mm}$ length SCF and two fiber balls structure, and the red line is the spectrum of the MZI with only $5 \mathrm{~mm}$ length SCF without fiber ball. It can be seen that the No. 1 MZI interference fringe has a visibility around $20 \mathrm{~dB}$ much more than No. 2 has. The coupling effects of fiber ball structure can help the interference enhancement of multi-core fiber (MCF). Figure 1(b) shows the transmission spectra of the proposed MZI with the lengths of $5 \mathrm{~mm}$ and $7 \mathrm{~mm}$ SCFs. The intensity of the interference light exhibits a certain periodicity as a function of wavelength, also it is shown that the sensor's free spectrum range increases as the SCF length $L$ decreases. The experimental results in Fig. 1(b) will be followed by detailed theoretical derivation. 


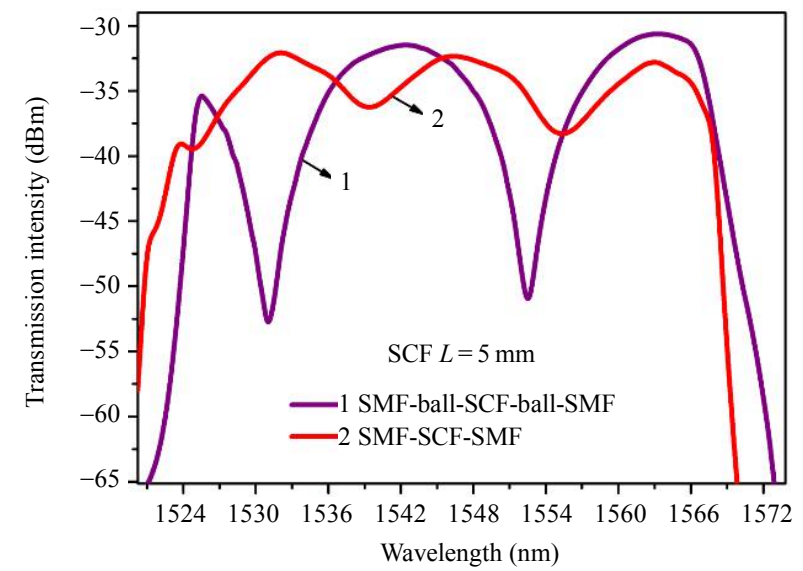

(a)

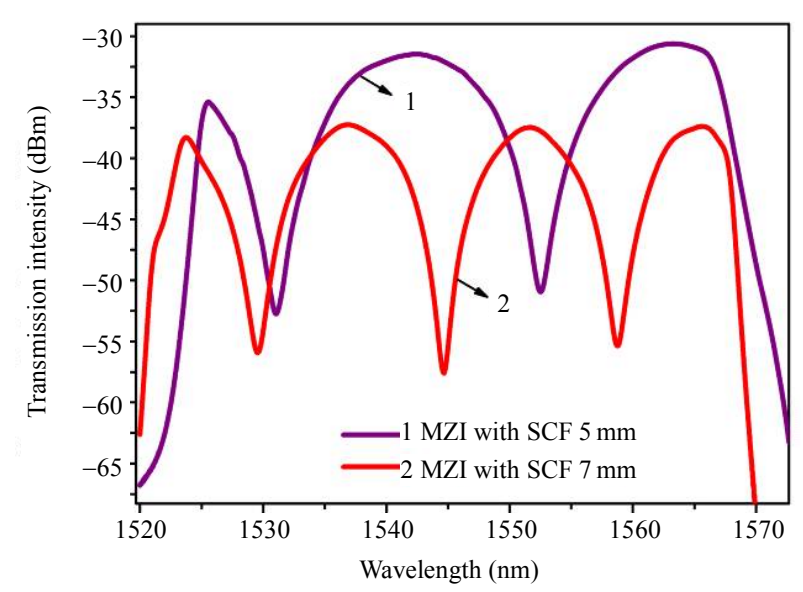

(b)

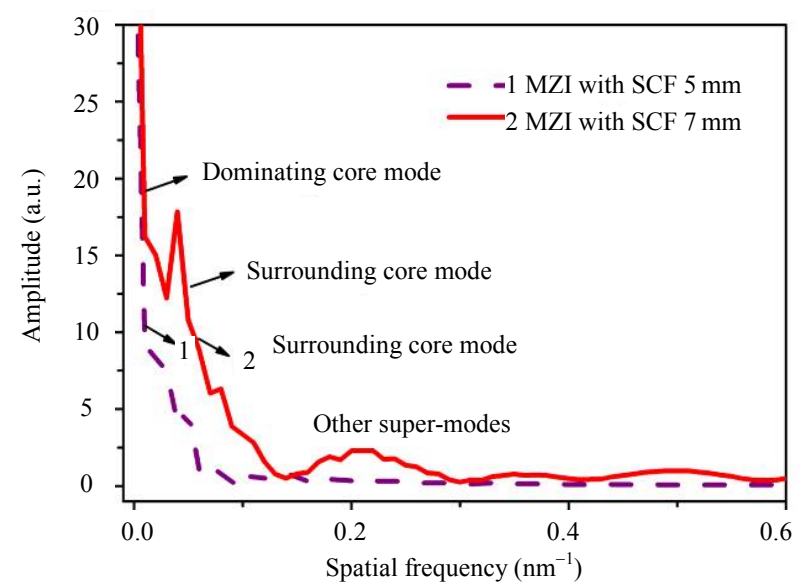

(c)

Fig. 1 Measured transmission spectra of the MZIs: (a) different MZI structures, (b) different lengths with two fiber balls, and (c) spatial frequency by FFT for the transmission spectra of the different MZI structures.

To understand clearly the number and the power distribution of the interference modes, the transmission spectrum [illustrated in Fig. 1(b)] is achieved by fast Fourier transform (FFT) to get the spatial frequency spectrum, as shown in Fig. 1(c). It can be observed that one interference peak appears in the spatial spectrum, which is formed due to the interference between the dominating core mode and the surrounding core modes. The dominated peak of the dotted line is situated at the frequency of $0.0501 \mathrm{~nm}^{-1}$, in which the solid line is $0.0400 \mathrm{~nm}^{-1}$. It is the fact that other super-modes are also possibly excited, which results in some smaller spatial frequency peaks.

As shown in Fig. 2, the light filed propagation and intensity distribution of the SMF-ball-SMFball-SMF [Fig. 2(a)] and SMF-ball-SCF-ball-SMF [Figs. 2(b)-2(d)] are simulated by beam propagation. In the simulation, lengths of SMF and SCF are maintained in the same of $7 \mathrm{~mm}$, and the diameters of balls are adjusted for $0.3 \mathrm{~mm}, 0.4 \mathrm{~mm}$, and $0.5 \mathrm{~mm}$, respectively. It can be seen from Fig. 2(a) that the intensity of the light field is mainly distributed in the central core and the coupling efficiency with light is low. In the SMF-ball-SCF-ball-SMF structure, the light can be better coupled from the lead-in SMF into the seven-core fiber because of the ball of SMF. As shown in Fig. 2(b), the intensity of the light field is not only distributed in the central core, but also distributed in other cores and cladding of the SCF when the diameter of ball is $300 \mu \mathrm{m}$. From Figs. 2(c) and 2(d), we can get that the change of single-mode fiber balls' diameter will have a certain impact on the output intensity of the light field.

From the simulation results, it can be concluded that the energy change and the final output energy are different when the diameters of the single-mode fiber balls in the middle section are different. According to this simulation, the diameters of the single-mode fiber balls which are suitable for this paper can be selected to obtain good coupling efficiency. By analyzing the three figures [Figs. 2(b)-2(d)], the intensity of the light field coupled from the fiber balls to the core of the output SMF is 
stronger when the diameter of the single-mode fiber balls is $400 \mu \mathrm{m}$. In this case, a better transmission spectrum can be formed which is more conducive to the analysis of experimental results. In the subsequent research process, the researchers could choose an appropriate size of the seven-core fiber and the single-mode fiber balls as an important part of the MZI sensor. (a)

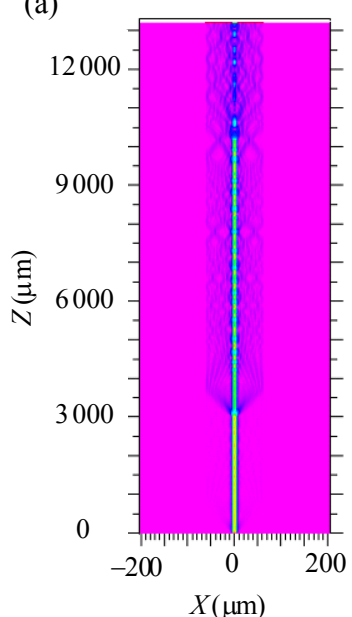

(c)

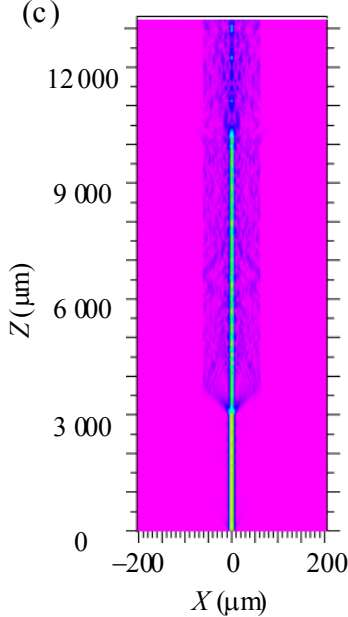

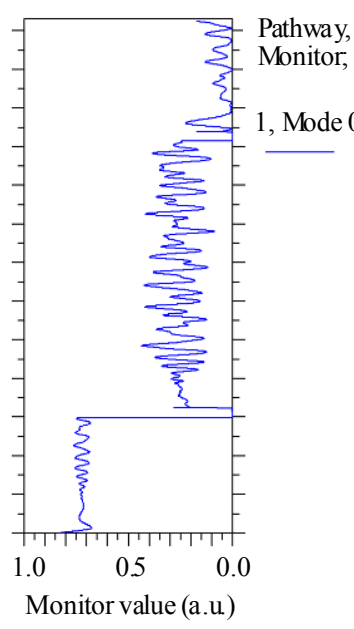
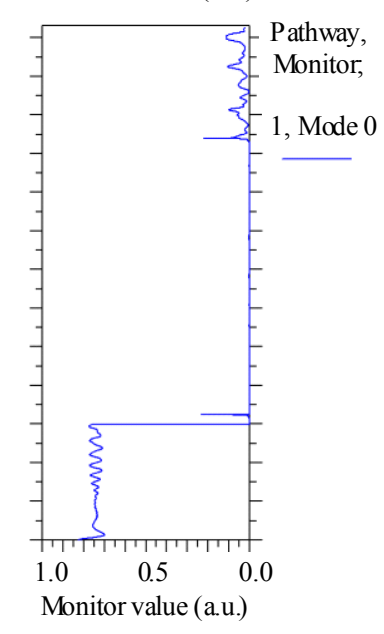

(b)

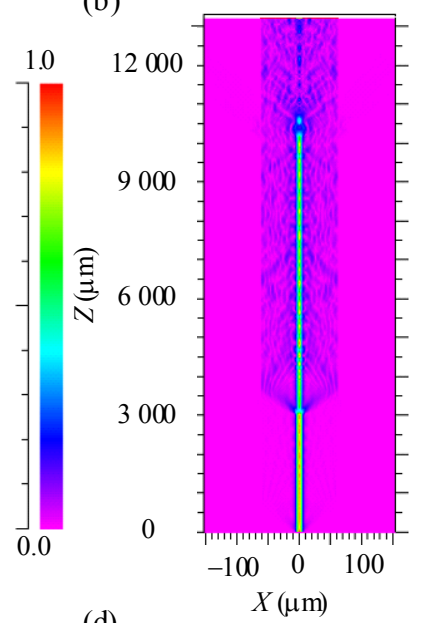

(d)

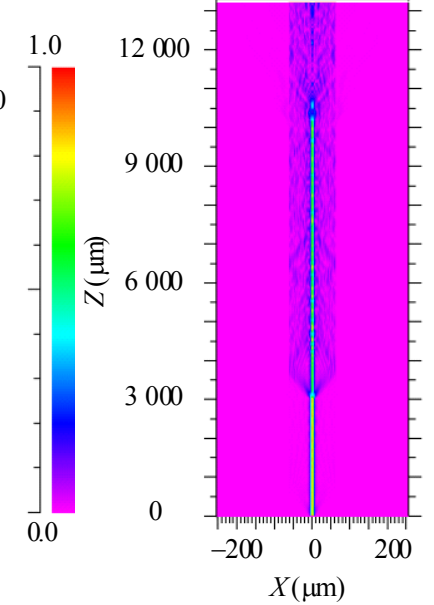

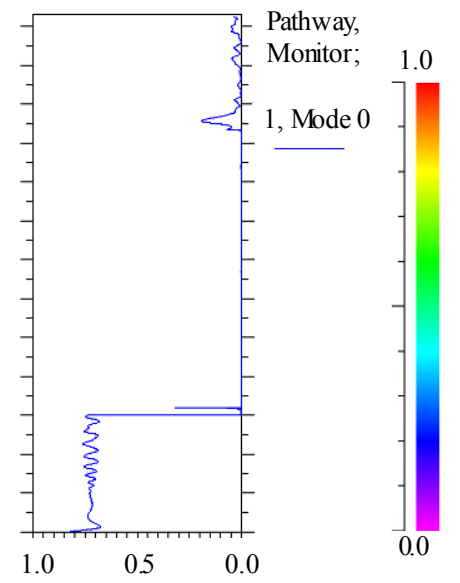

Monitor value (a.u.)

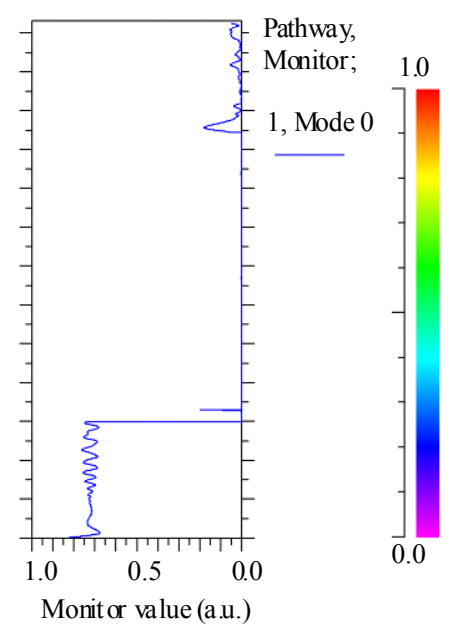

Fig. 2 Light propagation along the ball-SMF-ball and ball-SCF-ball structure: (a) the structure of SMF-ball-SMF-ball-SMF with the ball of $400 \mu \mathrm{m}$, (b) the structure of SMF-ball-SCF-ball-SMF with the ball of $300 \mu \mathrm{m}$, (c) the structure of SMF-ball-SCF-ball-SMF with the ball of $400 \mu \mathrm{m}$, and (d) the structure of SMF-ball-SCF-ball-SMF with the ball of $500 \mu \mathrm{m}$.

\subsection{Sensor fabrication}

The structure of the full fiber MZI sensor based on the SCF and the optical fiber fusion ball is shown in Fig. 3. The fabrication procedures are able to achieve by a glass processing and splicing system (AFL Fujikura, LZM-100 LaserMaster) in Ball Lens Menu, which is selected in the FPS software supporting the LZM-100. The splicing system uses a $\mathrm{CO}_{2}$ laser as the heating source to soften fibers due to its cleanness, low maintenance, and long-term consistency, which can ensure repeatable performance. The laser beam path of the fusion splicer is shown in Fig.3(a). The maximum power of the $\mathrm{CO}_{2}$ laser is $30 \mathrm{~W}$. After passing a safety shutter and a reflecting mirror, the laser beam is split into two paths to heat fiber on opposite sides with an angle about $170^{\circ}$ to avoid coupling, which ensures that the fiber transverse section can be heated uniformly. A shutter is used to modulate the laser. The fiber processing machine could show the hot images of the fiber and the information of the laser 
during the process by the charge coupled devices (CCDs) and the thermal piles. The beam path is adjusted by the mirrors mounted on the mechanical tunable fixtures. The remaining laser power is completely absorbed by 2 thermal piles for safety, measurement of power, beam size, and beam position. The beam power should be aligned to the fiber and ensure the camera for a good measurement accuracy. The fusion splicer has a pair of rotation motors mounted on the left and right translation motors respectively, which rotates with no restriction. A pair of fiber holders is fixed on the rotation motors. The fiber is homogeneously exposed to the laser from both sides of the fiber deflector. The formation of an MZI structure with a $\mathrm{CO}_{2}$ laser splicer is shown in Fig. 3(b).

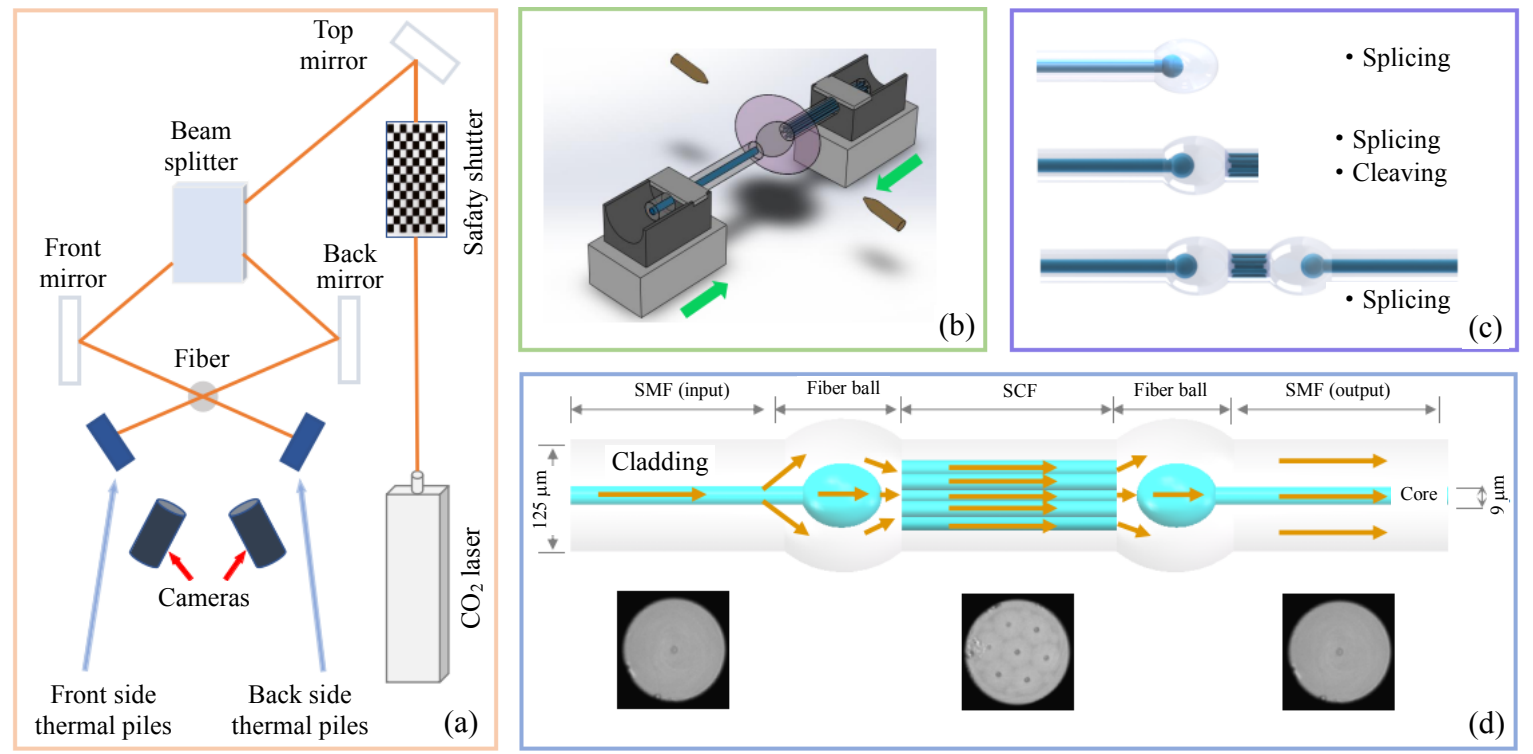

Fig. 3 Structure of the full fiber MZI sensor based on the SCF and the optical fiber fusion ball: (a) laser beam path of the fusion splicer, (b) formation of MZI structure, (c) processing program of MZI, and (d) sensing structure diagram.

We set the proposed MZI processing program, which consists of the following steps:

Step 1: The fiber balls used in the symmetrical structure here are prepared beforehand by splicing at one end of an SMF. As shown in Fig. 3(c), the fiber is fixed on the right holder, and the two beams of the $\mathrm{CO}_{2}$ laser are focused on the end of the fiber. Both the rotation and translation movements must operate synchronously. To obtain a specific diameter fiber ball, the translation speed should match with the rotation speed rationally. The rotation motor should twist $360^{\circ}$ at $150^{\circ} / \mathrm{s}$ when the translation motors run at $0.05 \mathrm{~mm} / \mathrm{s}$ feeding speed. The fiber ball structure about $400 \mu \mathrm{m}$ diameter is obtained.

Step 2: Cleave the end of seven-core fiber and splice the flat end with the fiber ball [Fig. 3(d)]. Then cleave the other end of seven-core fiber again and make a section of $7 \mathrm{~mm}$ length SCF retained.

Step 3: Splice the structure fabricated at Step 2 with another fiber ball, forming "SMF-ball-SCF-ball-the SMF" optical waveguide, then the in-line fiber MZI structure is completed, as shown in Fig. 3(d).

The parameters of the program for fabricating the MZI structure are listed in Table 1.

Table 1 Parameters of the program for fabricating the MZI structure.

\begin{tabular}{cc}
\hline Parameters & Value \\
\hline Fiber diameter & $125 \mu \mathrm{m}$ \\
Ball diameter & $400 \mu \mathrm{m}$ \\
Strip length & $17 \mathrm{~mm}$ \\
Pre-heat time & $5 \mathrm{~s}$ \\
Lens creation power & $762 \mathrm{bits}$ \\
Lens type & Cylindrical lens \\
Rotator speed & $150 \mathrm{deg} / \mathrm{s}$ \\
Feeding speed & $0.05 \mathrm{~mm} / \mathrm{s}$ \\
\hline
\end{tabular}




\subsection{Sensing principle}

In the SCF, the optical path differences are due to the effective refractive index differences among the central core mode, the surrounding core mode, and the cladding modes. Generally, each optical path difference induced by the combination of these modes could generate either interference weak or strong interference (depending on the intensity of the involved modes). Here, we only consider the dominant interference caused by the optical path difference (OPD) induced by the central core mode and the surrounding core mode of the MCF [16], so this structure still can be regarded as an MZI model [17], then the transmission spectrum intensity of the proposed interferometer can be explained as

$$
I=I_{c}+I_{s}+2 \sqrt{I_{c} I_{s}} \cos \Delta \Phi
$$

where $I_{c}$ is the central core mode intensity, $I_{s}$ is the surrounding core mode intensity of the SCF, and $\Delta \Phi$ is the phase difference between the core mode and the surrounding core mode, which can be defined as

$$
\Delta \varphi=\frac{2 \pi \Delta n_{\mathrm{eff}} L}{\lambda_{0}}
$$

where $\lambda_{0}$ is the wavelength of the propagating light, $\Delta n_{\text {eff }}$ is the effective refractive index (ERI) coefficient difference between the core mode and the surrounding core mode, and $L$ is the interaction length of SCF between the ball-shape fiber structures, namely, the length of interferometer arm. If phase difference satisfies the condition $\Delta \Phi=(2 m+$ 1) $\pi, m=1,2,3, \cdots$, the intensity of transmission reaches its valley value at the wavelength as follows:

$$
\lambda_{m}=\frac{2 \Delta n_{\mathrm{eff}} L}{2 m+1} .
$$

The initial transmission spectrum of the interference structure acquired by a wide spectrum source and spectrometer is shown in Fig. 4.

The length of interferometer arm and the ERI of the $m$-order attenuation peak would be changed by the external environment parameters, such as temperature, strain, and refractive index, which further brings about shifts of the interference fringes. So, the measured value can be determined by monitoring the transmission spectrum.

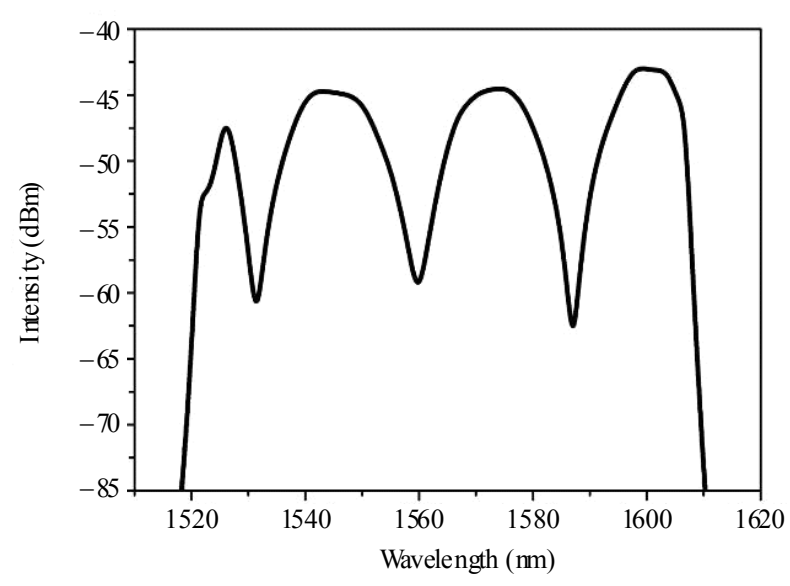

Fig. 4 Initial transmission spectrum.

Also from (3), the free spectral range (FSR) of the interference fringe pattern is determined by the optical path difference, $\Delta n_{\text {eff }} L$, which can be approximated by [18] follows:

$$
\begin{aligned}
F S R & =\left|\lambda_{m}-\lambda_{m-1}\right|=\frac{4 \Delta n_{\mathrm{eff}} L}{(2 m+1)(2 m-1)} \\
& =\frac{\lambda_{m}^{2}}{\Delta n_{\mathrm{eff}} L} \cdot \frac{2 m+1}{2 m-1} \approx \frac{\lambda_{m}^{2}}{\Delta n_{\mathrm{eff}} L} .
\end{aligned}
$$

The FSR determines the density of the comb-like transmission spectrum. It can be seen that the FSR will decrease with interferometer length $L$.

Figure 5 shows the schematic structure of the microdisplacement measurement device. According to the principle of leverage, the actual lengths of the lever arms are $l_{4}$ and $l_{5}$. When the movable arm turns around an angle of $2 \Delta \theta$ while the fixed arm remains stationary, it can be equivalent to the two arms rotating at an angle of $\Delta \theta$ at the same time, as shown by the blue line in Fig. 5(d).

The right triangle is constructed, and three equations, $C=\theta_{10}-\theta_{20}$ ( $C$ is a constant), $\theta_{1}=\theta_{10}+\Delta \theta$, and $\theta_{2}=\theta_{20}+\Delta \theta$ are defined, where $\theta_{1}$ is the angle between $l_{4}$ and the central axis, $\theta_{2}$ is the angle between $l_{5}$ and the central axis, and $\theta_{10}$ and $\theta_{20}$ are the initial states of $\theta_{1}$ and $\theta_{2}$, respectively. And $\Delta \theta$ is the angle of the arms turned. 
We can easily get $\sin \theta_{1}=h / 2 l_{4}$ and $l=2 l_{5} \sin \theta_{2}$ through the mathematical analysis of Fig. 5(d).
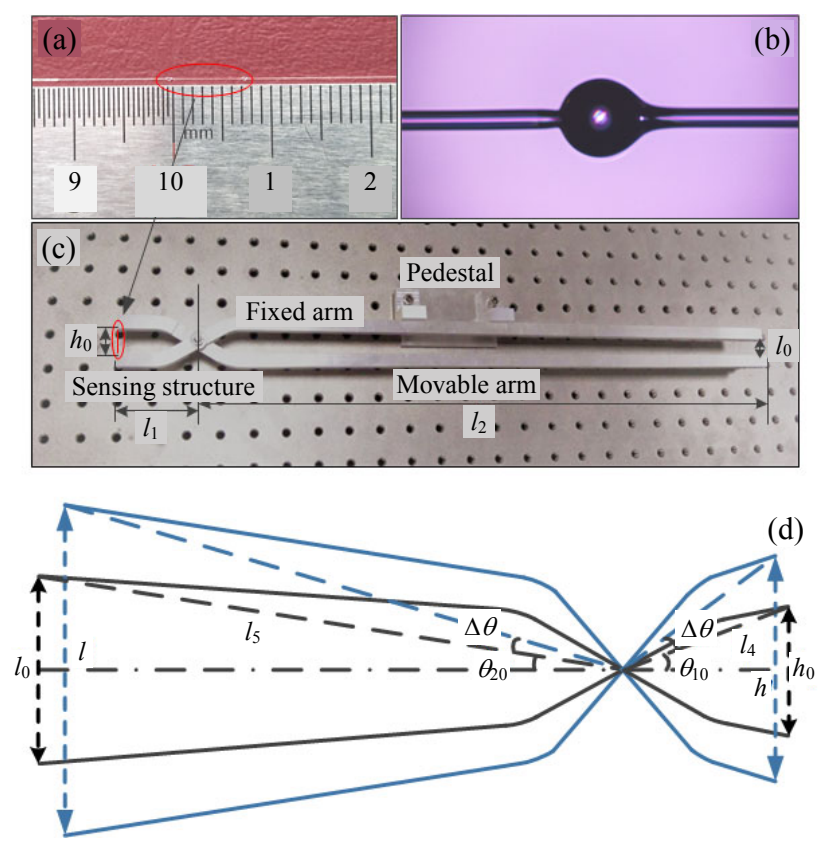

Fig. 5 Schematic structure of the microdisplacement measurement device: (a) sensing structure, (b) melting ball physical map, (c) the side sectional view of the microdisplacement measurement device, and (d) schematic structure of the micro-displacement measurement device.

Then (5) is achieved by substituting $C=\theta_{10}-\theta_{20}$, $\theta_{1}=\theta_{10}+\Delta \theta, \theta_{2}=\theta_{20}+\Delta \theta$, and $\sin \theta_{1}=h / 2 l_{4}$ into $l=$ $2 l_{5} \sin \theta_{2}$.

$$
l=\frac{l_{5} \cos C}{l_{4}} h-\frac{2 l_{5} \sin C}{l_{4}} \sqrt{l_{4}^{2}-(h / 2)^{2}} .
$$

In the microdisplacement experiment, $l$ changes from the initial value $l_{0}$ with a step of $0.12 \mathrm{~mm}$. The overall variation of $l$ is $1.08 \mathrm{~mm}$, in which the microdisplacement at the other end of the arm is so small compared with $l_{4}(5.85 \mathrm{~cm})$ that $\sqrt{l_{4}^{2}-(h / 2)^{2}}$ can be regarded as a fixed value $\sqrt{l_{4}^{2}-\left(h_{0} / 2\right)^{2}}$. Then, (5) can be written as (6) as follows:

$$
l=\frac{l_{5} \cos C}{l_{4}} h-\frac{2 l_{5} \sin C}{l_{4}} \sqrt{l_{4}^{2}-\left(h_{0} / 2\right)^{2}} .
$$

Therefore, $l$ rises in approximate linearity with an increase in $h$ in the experimental range. Assuming that the elongation of the micrometer caliper is $\Delta l$, the arc length swept by the arms can be nearly regarded as the chord length. The equation
$\Delta h=\frac{l_{4}}{l_{5} \cos C} \Delta l$ can be easily got. The axial microdisplacement $\varepsilon$ in the sensor part can be calculated from (6) and shown in (7) as follows:

$$
\varepsilon=\frac{\Delta h}{h_{0}}=\frac{l_{4}}{l_{5} h_{0} \cos C} \Delta l .
$$

When the ambient temperature of the optical fiber changes, the sensitivity of the characteristic wavelength of the valley in the transmission spectrum can be expressed as [19]

$$
\frac{\partial \lambda_{m}}{\partial T}=\frac{2}{2 m+1}\left[\Delta n_{\mathrm{eff}} \frac{\partial L}{\partial T}+L\left(\frac{\partial n_{\mathrm{co}}^{\mathrm{eff}}}{\partial T}-\frac{\partial n_{\mathrm{cl}, j}^{\mathrm{eff}}}{\partial T}\right)\right]
$$

where $\Delta n_{\text {eff }}$ represents the effective refractive index between the fiber core module and the $j$-order cladding module, and $\Delta n_{\mathrm{eff}}=n_{\mathrm{co}}^{\text {eff }}-n_{\mathrm{cl}, j}^{\text {eff }}, T$ indicates temperature.

When the axial microdisplacement $\varepsilon$ of the fiber is changed, the sensitivity of the characteristic wavelength of the valley in the transmission spectrum can be expressed as follows [19]:

$$
\frac{\partial \lambda_{m}}{\partial \varepsilon}=\frac{2}{2 m+1}\left[\Delta n_{\mathrm{eff}} \frac{\partial L}{\partial \varepsilon}+L\left(\frac{\partial n_{\mathrm{co}}^{\mathrm{eff}}}{\partial \varepsilon}-\frac{\partial n_{\mathrm{cl}, j}^{\mathrm{eff}}}{\partial \varepsilon}\right)\right] .
$$

From (8) and (9), the wavelength value of the wave valley in the interference spectrum is in the different proportion to the changes of temperature and microdisplacement, that is, the response sensitivities to temperature and microdisplacement are different. Therefore, temperature and microdisplacement can be measured simultaneously by the wavelength shift of the valley of the transmission spectrum and the sensitivity coefficient matrix.

\section{Experiment}

The experimental system is shown in Fig. 6, including a light source with a flat output in the range from $1516.30 \mathrm{~nm}$ to $1616.30 \mathrm{~nm}$ and an OSA (AQ6370C) with a wavelength resolution of $0.05 \mathrm{~nm}$. In the application of Mach-Zehnder interference sensor, there is always a problem of cross sensitivity. When sensing, it is difficult to distinguish the changes of measured quantities caused by 
microdisplacement and temperature. Therefore, the temperature characteristic of the sensor structure should be analyzed before the microdisplacement experiment.

\subsection{Temperature characteristic experiment}

For the temperature characteristic experiment, the sensor head is placed into the thermostatic heater plate and then the temperature is allowed to rise from $27.9{ }^{\circ} \mathrm{C}$ to $119.5{ }^{\circ} \mathrm{C}$. The temperature characteristic test platform is shown in Fig. 6. The shifts of the two dips are recorded about $10{ }^{\circ} \mathrm{C}$. The output spectra and temperature response characteristics curve are shown in Fig. 7.

From Fig. 7(a), both the dip shifts to the long wavelength with temperature rising and the shift of Dip 1 and Dip 2 are more intuitive. As can be seen from Fig. 7, compared with the change of light intensity, the linearity of the drift amount of the wavelength is better, so the drift amount will be analyzed next. As shown in Fig.7(b), Dip 1 moves from $1559.7580 \mathrm{~nm}$ to $1568.7939 \mathrm{~nm}$, with a sensitivity of $98.65 \mathrm{pm} /{ }^{\circ} \mathrm{C}$, meanwhile, Dip 2 moves from $1586.9838 \mathrm{~nm}$ to $1595.2024 \mathrm{~nm}$, with a sensitivity of $89.72 \mathrm{pm} /{ }^{\circ} \mathrm{C}$.

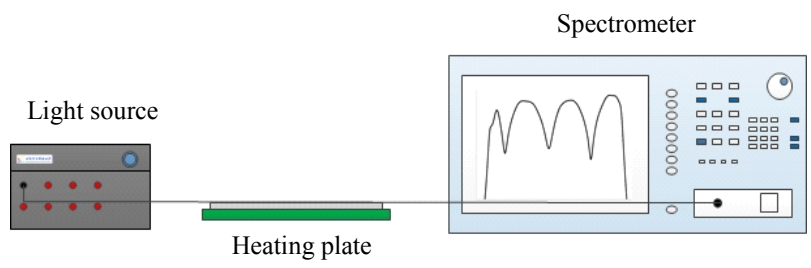

Fig. 6 Temperature characteristic test platform.

\subsection{Microdisplacement experiment}

The experimental setup for the microdisplacement measurement is shown in Fig. 5(a). The lever structure is made by aluminum. The sensing structure is pasted to the end of the short arms with epoxy resin named DP420. Based on the laboratory experience and characteristic of the sensor, DP420 epoxy resin glue, a kind of adhesive agent with good insulating properties, small after curing shrinkage shape variables, and high mechanical strength, is chosen. Usually, to be solidified, it needs over 12 hours under room temperature or shorter by heating. The sensor structure is initially in a natural straightening state.

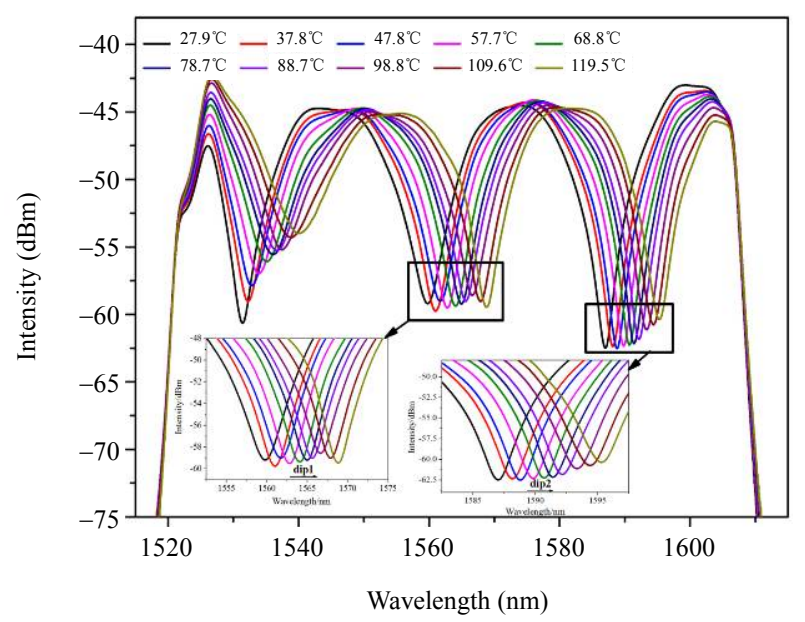

(a)

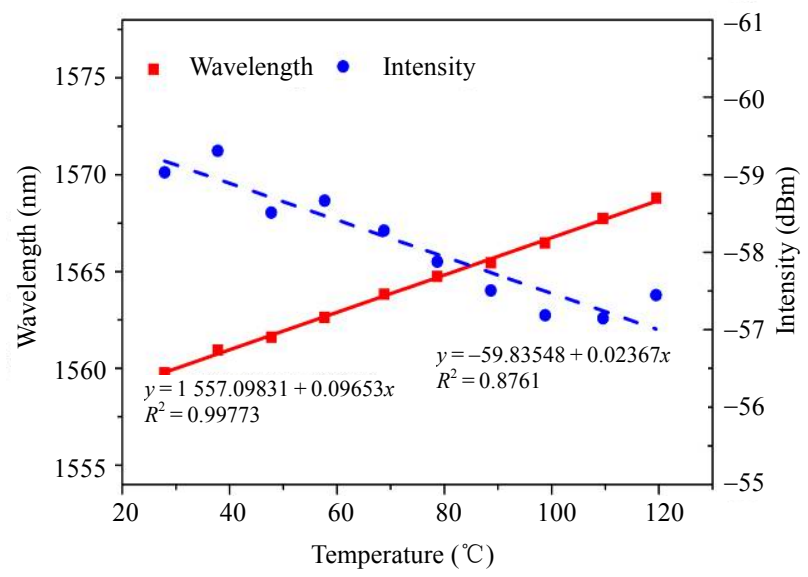

(b)

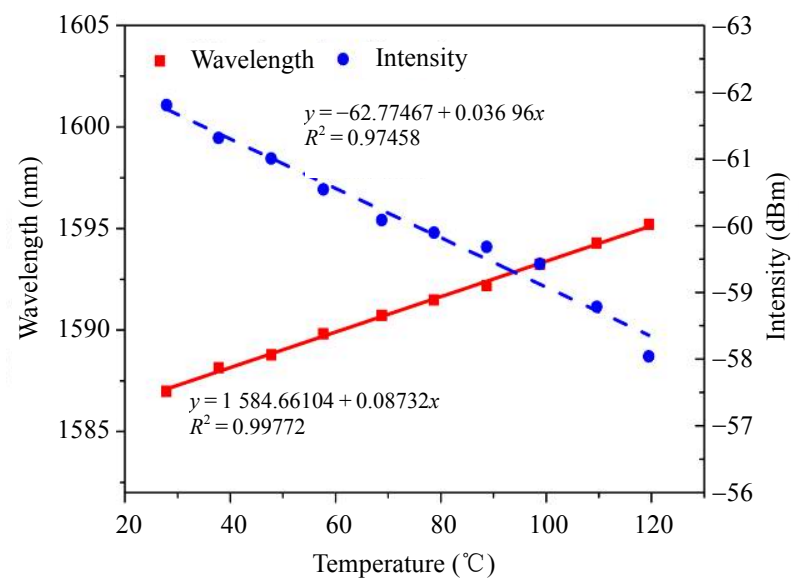

(c)

Fig. 7 Temperature characteristic experiment results: (a) experimental transmission spectra with different temperatures, (b) relationship between temperature and wavelength shift of Dip 1, and (c) relationship between temperature and wavelength shift of Dip 2. 


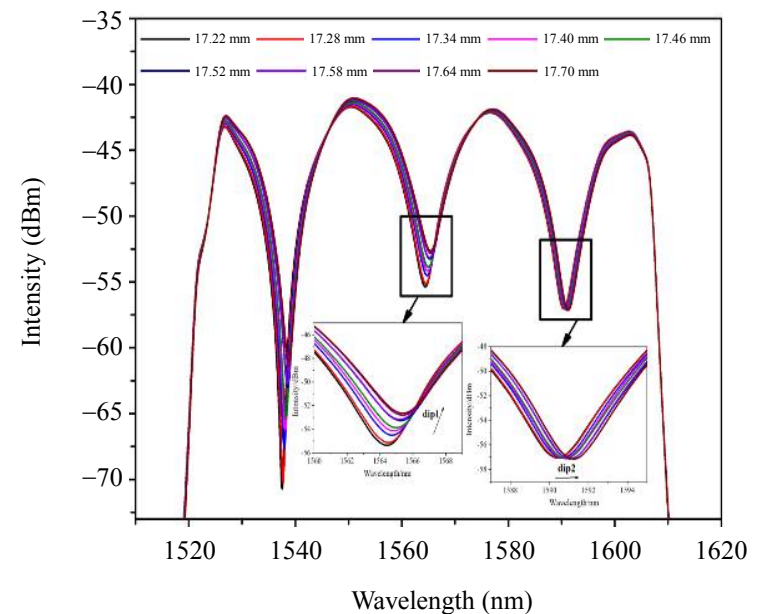

(a)

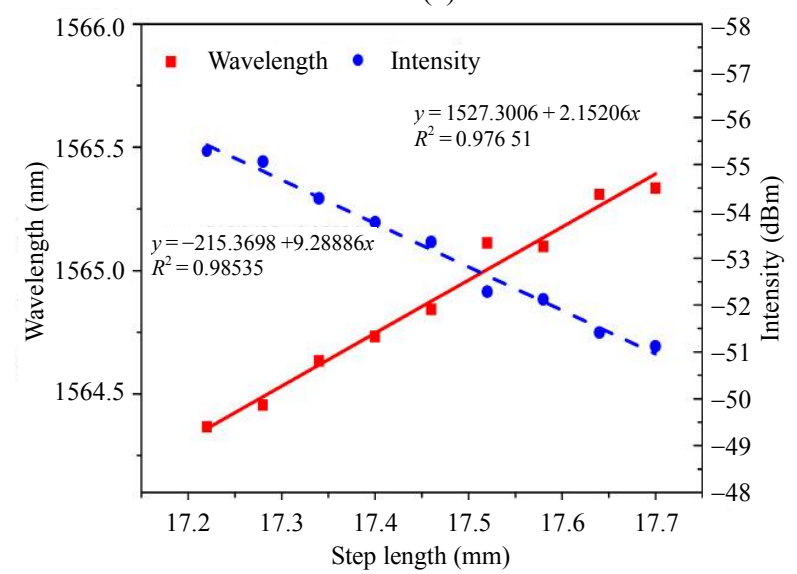

(b)

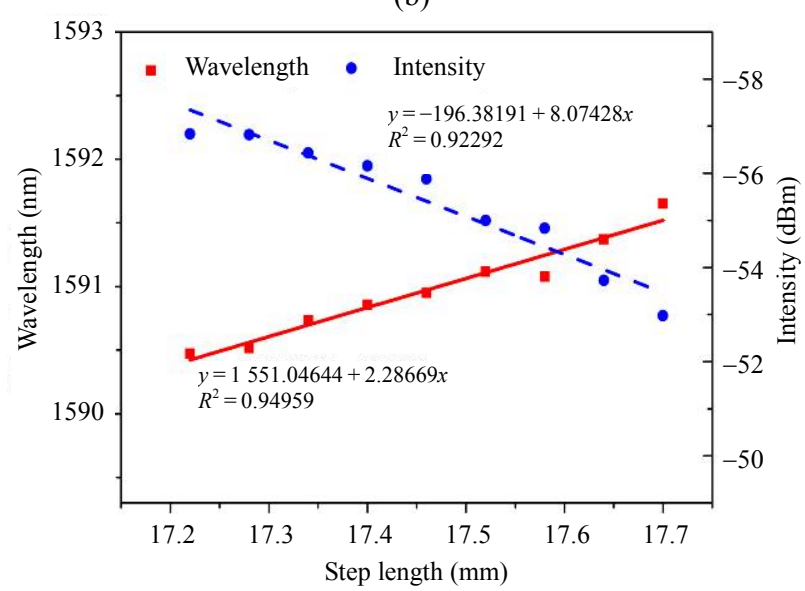

(c)

Fig. 8 Microdisplacement experimental results: (a) experimental transmission spectra with different step lengths, (b) relationship between step length and wavelength shift of Dip 1, and (c) relationship between step length and wavelength shift of Dip 2.

The microdisplacement experiment is carried out at room temperature. In order to guarantee the service life of the sensor due to excessive tensile fracture and prevent the sensor structure, the range of measuring is from $17.22 \mathrm{~mm}$ to $17.70 \mathrm{~mm}$, and the step length is $0.06 \mathrm{~mm}$ (using vernier caliper to control the displacement and the precision of the vernier caliper is $0.02 \mathrm{~mm}$ ). The output spectra and microdisplacement response characteristics curve are shown in Fig. 8.

By increasing the distance of the vernier caliper, the transmission spectrum produces a corresponding wavelength shift. Compared with the change of light intensity, the linearity of the drift amount of the wavelength is better. From Fig. 8(b), we can see that the microdisplacement sensitivities of Dip 1 and Dip 2 are $2017.71 \mathrm{pm} / \mathrm{mm}$ and $2457.92 \mathrm{pm} / \mathrm{mm}$, respectively.

\subsection{Results and discussion}

It could be concluded that the microdisplacement and temperature sensitivities of the two dips are different from the above experimental results. The relationship between the shifts of Dip 1, Dip 2, temperature, and microdisplacement can be expressed in matrix form as

$$
\left[\begin{array}{l}
\Delta \lambda_{B 1} \\
\Delta \lambda_{B 2}
\end{array}\right]=\left[\begin{array}{ll}
K_{T 1} & K_{l 1} \\
K_{T 2} & K_{l 2}
\end{array}\right]\left[\begin{array}{c}
\Delta T \\
\Delta l
\end{array}\right]
$$

where $\Delta \lambda_{B 1}$ and $\Delta \lambda_{B 2}$ are the shifts of the Dip 1 and Dip 2, respectively, $\Delta T$ is the change of temperature, $\Delta h$ is the value of microdisplacement, $K_{T 1}$ and $K_{l 1}$ are the temperature and microdisplacement sensitivities of Dip 1, and $K_{T 2}$ and $K_{l 2}$ are the temperature and microdisplacement sensitivities of Dip 2.

All the sensitivity coefficients can be obtained from the experimental results. We can use a sensitivity matrix to obtain the variation of temperature and microdisplacement. The relationship is given as

$$
\left[\begin{array}{c}
\Delta T \\
\Delta l
\end{array}\right]=\frac{1}{D}\left[\begin{array}{cc}
K_{l 2} & -K_{l 1} \\
-K_{T 2} & K_{T 1}
\end{array}\right]\left[\begin{array}{l}
\Delta \lambda_{B 1} \\
\Delta \lambda_{B 2}
\end{array}\right]
$$

where $D=K_{T 1} K_{l 2}-K_{T 2} K_{l 1}$.

Then substitute the experimental data into (10) 
and get the following equation:

$$
\left[\begin{array}{c}
\Delta T \\
\Delta l
\end{array}\right]=\frac{1}{4.9 \times 10^{8}}\left[\begin{array}{cc}
2457.92 & -2017.71 \\
-89.72 & 98.65
\end{array}\right]\left[\begin{array}{l}
\Delta \lambda_{B 1} \\
\Delta \lambda_{B 2}
\end{array}\right] .
$$

The sensor structure is subject to external factors (such as temperature and microdisplacement) and the transmission spectrum causes a corresponding wavelength shift. Temperature and microdisplacement can be further obtained by obtaining the corresponding wavelength drift of the transmission spectrum trough (i.e., $\Delta \lambda_{B 1}$ and $\Delta \lambda_{B 2}$ ), thereby the measurement of temperature and microdisplacement can be achieved simultaneously.

It can be known from (11) that if the sensitivities of the fiber sensor to temperature and microdisplacement changes are known, the wavelength change of the transmission spectrum can be monitored by a spectrometer and substituted into (11) for calculation. In the actual solution calculation, the difference in sensitivity coefficient between the two sets of fiber structures is greater and the sensing of temperature and microdisplacement is more reliable.

Compared with the early MZI structure [17-19], the proposed sensor in this paper has a shorter structure and deeper interference valley. In terms of the manufacturing process of different MZI structures, the proposed SMF-ball-SCF-ball-SMF sensor structure has a simpler manufacturing process and the length of the MZI is more controllable. Compared with the tapering MZI sensor [17], the proposed sensor has better reliability and strength.

\section{Conclusions}

In this paper, an all-fiber MZI sensor based on a seven-core fiber and two single-mode fiber balls is proposed and investigated for simultaneously measuring temperature and microdisplacement.

The experimental results show that under the external environment, the MZI interference structure in the paper changes the output light intensity, and the transmission spectrum causes a corresponding wavelength shift. The sensitivity of the sensor for the temperature of the two dips are $98.65 \mathrm{pm} /{ }^{\circ} \mathrm{C}$ and $89.72 \mathrm{pm} /{ }^{\circ} \mathrm{C}$, respectively. And the sensitivities of the sensor for the microdisplacement are $2017.71 \mathrm{pm} / \mathrm{mm}$ and $2457.92 \mathrm{pm} / \mathrm{mm}$, respectively.

Compared with other interferometric sensors of the MZI structure, this structure has advantages of simple production process, high reliability, and better strength. The sensor will have broad applications in the field of automation equipment, robots, and the management of roads and bridges.

\section{Acknowledgment}

This work was supported by the Program for Changjiang Scholars and Innovative Research Team in University (Grant No. IRT_16R07), the Importation and Development of High-Caliber Talents Project of Beijing Municipal Institutions (Grant No. IDHT20170510), the 111 Project (Grant No. D17021), and Research Project of Beijing Education Committee (Reseach on all-in-fiber integrated MZI sensor fabricated by $\mathrm{CO}_{2}$ laser).

Open Access This article is distributed under the terms of the Creative Commons Attribution 4.0 International License (http://creativecommons.org/licenses/by/4.0/), which permits unrestricted use, distribution, and reproduction in any medium, provided you give appropriate credit to the original author(s) and the source, provide a link to the Creative Commons license, and indicate if changes were made.

\section{References}

[1] A. Babu and B. George, "Design and development of a new non-contact inductive displacement sensor," IEEE Sensors Journal, 2018, 18(3): 976-984.

[2] S. Rana, B. George, and V. J. Kumar, "An efficient digital converter for a non-contact inductive displacement sensor," IEEE Sensors Journal, 2018, 18(1): 263-272.

[3] X. Rimpault, E. B. Nehme, M. Balazinski, and J. R. R. Mayer, "Online monitoring and failure detection of capacitive displacement sensor in a Capball device using fractal analysis," Measurement, 2018, 118: 23-28.

[4] A. Arifin, A. M. Hatta, Sekartedjo, M. S. Muntini, and 
A. Rubiyanto, "Long-range displacement sensor based on SMS fiber structure and OTDR," Photonic Sensors, 2015, 5(2): 166-171.

[5] M. Sethuramalingam and U. Subbiah, "Enhancing the linearity characteristics of photoelectric displacement sensor based on extreme learning machine method," Photonic Sensors, 2015, 5(1): 24-31.

[6] X. L. Xie, B. W. Wang, L. L. Zhou, L. Weng, and Y. Sun, "Research on torsional ultrasonic attenuation characteristics of the magnetostrictive displacement sensor waveguide," Diangong Jishu Xuebao/Transactions of China Electrotechnical Society, 2018, 33(3): 689-696.

[7] S. M. Chen, Y. Liu, X. X. Liu, Y. Zhang, and W. Peng, "Self-compensating displacement sensor based on hydramatic structured transducer and fiber Bragg grating," Photonic Sensors, 2015, 5(4): 351-356.

[8] C. Zhong, C. Y. Shen, J. L. Chu, X. Zou, K. Li, Y. X. Jin, et al., "A displacement sensor based on a temperature-insensitive double trapezoidal structure with fiber Bragg grating," IEEE Sensors Journal, 2012, 12(5): 1280-1283.

[9] C. W. Shangguan, W. He, W. Zhnag, F. Luo, and L. Q. Zhu, "Optical fiber Fabry-Perot displacement sensor based on chemical etching method," Semiconductor Optoelectronics, 2018, 39(02): 170-173.

[10] C. Liu, W. Zhang, M. L. Dong, X. P. Lou, and L. Q. Zhu, "Dual-parameter sensing characteristics of long period fiber grating cascaded with fiber MZ structure fabricated by $\mathrm{CO}_{2}$ laser," Infrared \& Laser Engineering, 2017, 46(9): 922001-922007.

[11] Y. Wang, Y. H. Li, C. R. Liao, D. N. Wang, M. W. Yang, and P. X. Lu, "High-temperature sensing using miniaturized fiber in-line Mach-Zehnder interferometer," IEEE Photonics Technology Letters, 2009, 22(1): 39-41.
[12] H. S. Lin, Y. M. Raji, J. H. Lim, S. K. Liu, M. R. Mokhtar, and Z. Yusoff, "Packaged in-line Mach-Zehnder interferometer for highly sensitive curvature and flexural strain sensing," Sensors \& Actuators A: Physical, 2016, 250: 237-242.

[13] J. P. Chen, J. Zhou, and Z. H. Jia, "High-sensitivity displacement sensor based on a bent fiber Mach-Zehnder interferometer," IEEE Photonics Technology Letters, 2013, 25(23): 2354-2357.

[14] C. Y. Shen, J. L. Chu, Y. F. Lu, D. B. Chen, C. Zhong, Y. Li, et al., "High sensitive micro-displacement sensor based on M-Z interferometer by a bowknot type taper," IEEE Photonics Technology Letters, 2013, 26(1): 62-65.

[15] S. Zhou, B. Huang, and X. W. Shu, "A multi-core fiber based interferometer for high temperature sensing," Measurement Science \& Technology, 2017, 28(4): 045107.

[16] L. Duan, P. Zhang, M. Tang, R. X. Wang, Z. Y. Zhao, S. N. Fu, et al., "Heterogeneous all-solid multicore fiber based multipath Michelson interferometer for high temperature sensing," Optics Express, 2016, 24(18): 20210-20217.

[17] B. Yin, Y. Li, Z. B. Liu, S. C. Feng, Y. L. Bai, Y. Xu, et al., "Investigation on a compact inline multimode-single-mode-multimode fiber structure," Optics \& Laser Technology, 2016, 80: 16-21.

[18] L. C. Li, L. Xia, Z. H. Xie, and D. M. Liu, "All-fiber Mach-Zehnder interferometers for sensing applications," Optics Express, 2012, 20(10): 11109-11120.

[19] D. Wu, T. Zhu, K. S. Chiang, and M. Deng, "All single-mode fiber Mach-Zehnder interferometer based on two peanut-shape structures," Journal of Lightwave Technology, 2012, 30(5): 805-810. 